\title{
Le libéralisme insurrectionnel espagnol (1814-1830)
}

Irène Castells Olivan

\section{(2) OpenEdition \\ Journals}

Édition électronique

URL : https://journals.openedition.org/ahrf/1736

DOI : 10.4000/ahrf.1736

ISSN : 1952-403X

Éditeur :

Armand Colin, Société des études robespierristes

Édition imprimée

Date de publication : 1 juin 2004

Pagination : 221-233

ISSN : 0003-4436

\section{Référence électronique}

Irène Castells Olivan, « Le libéralisme insurrectionnel espagnol (1814-1830) ». Annales historiques de la Révolution française [En ligne], 336 | avril-juin 2004, mis en ligne le 15 juillet 2007, consulté le 22 avril 2022. URL : http://journals.openedition.org/ahrf/1736 ; DOI : https://doi.org/10.4000/ahrf.1736

Ce document a été généré automatiquement le 22 avril 2022.

Tous droits réservés 


\title{
Le libéralisme insurrectionnel espagnol (1814-1830) ${ }^{1}$
}

\author{
Irène Castells Olivan
}

1 Depuis la vague révolutionnaire déchaînée durant la dernière décennie du XVIIIe siècle par la grande Révolution de 1789 , et après le repli politique et organisationnel subi par le mouvement populaire et révolutionnaire depuis la fin de la Convention en France, les révolutions de 1820 parviennent à remettre au premier plan l'idée d'une révolution européenne.

2 Ces révolutions répondaient à un type révolutionnaire forgé en Espagne, ce qui permet de parler de "libéralisme insurrectionnel espagnol ", lequel renvoie au modèle des luttes contre l'absolutisme utilisées par les libéraux de l'Europe de la Restauration pour imposer aux monarques absolus le système constitutionnel. La formule subversive employée répondait à la théorie de l'insurrection par la voie du "pronunciamiento", selon laquelle l'insurrection nationale se produirait comme une simple conséquence du «pronunciamiento», un acte et un geste d'une personnalité militaire assumant la direction du soulèvement en lisant un Manifeste; il s'agissait d'un rite prévu pour provoquer l'adhésion des foyers libéraux qui attendraient clandestinement dans chaque localité le moment de se lancer dans la rue, de proclamer la Constitution et d'obtenir ainsi un changement de régime. Le pronunciamiento était par conséquent le paroxysme d'une conspiration de civils et de militaires, empreints du même idéal politique et constitutionnel, et il impliquait une véritable stratégie politique en vue de la prise de pouvoir. Bien qu'il résulte de l'expérience de la résistance espagnole aux Français entre 1808 et 1814 , il prit forme au cours des tentatives d'insurrections de caractère libéral qui se produisirent en Espagne entre 1814 et 1820.

L'apparition d'un archétype révolutionnaire

3 Pour comprendre la portée de ce modèle insurrectionnel, il faut le situer dans un contexte d'involution au niveau de la politique, caractérisant les années qui succédèrent à la défaite de Napoléon. Cette stratégie de lutte aurait été impossible sans l'expérience de la Révolution française et de l'épisode napoléonien. Dans ce sens, il ne faut pas oublier qu'après la défaite des sans-culottes à Paris, en avril-mai 1795, le 
mouvement populaire devint défensif et par conséquent conspirateur; il culmina avec l'insurrection frustrée organisée par la conjuration des Égaux. Pour assurer son succès, Babeuf considérait indispensable l'appui des militaires qu'il avait organisés parmi les secteurs jacobins de l'armée, mais qui furent aussi démantelés. Les Français assistèrent à son échec ainsi qu'à celui du régime du Directoire jusqu'à ce qu'ils se rendissent compte de la force apportée par l'armée pour se maintenir au pouvoir et stabiliser la Révolution, mais sur des principes très différents de ceux avec lesquels tout avait commencé, contrairement à ce qu'avait proclamé Napoléon. Ces événements exercèrent une grande influence sur les modèles insurrectionnels de l'Europe de la Restauration, parmi lesquels les pronunciamientos libéraux espagnols occupaient un premier plan. Le caractère despotique du régime bonapartiste s'accrut alors que la spécificité du cas espagnol signifia un changement important dans les dispositions de l'armée napoléonienne : à l'expérience dictatoriale de celle-ci s'ensuivit l'expérience libérale des pronunciamientos qui se produisirent en Espagne entre 1814 et 1830 et dont l'objectif n'était pas d'imposer une autorité militaire, mais d'implanter à nouveau la constitution de Cadix de $1812^{2}$, abolie par la restauration de Ferdinand VII en 1814. À travers le pronunciamiento en faveur de la Constitution, l'armée assumait le rôle de dépositaire de la volonté nationale et des aspirations libérales, devenant porte-parole des garanties politiques. Les conflits incessants entre le pouvoir civil et le pouvoir militaire qui se produisirent pendant la guerre contre Napoléon ouvrirent la voie à une lutte unitaire contre l'absolutisme de la part des militaires et des civils depuis 1814 pour la défense de la liberté et éviter les dangers du despotisme militaire ${ }^{3}$. Les origines historiques du phénomène doivent être cherchées dans la guerre contre Napoléon de 1808-1814.

4 Au cours de ces années, la victoire des Espagnols sur l'envahisseur français ne se définit pas seulement comme une irruption des masses, considérées comme des protagonistes, pour la première fois dans l'histoire, dans la vie politique et sociale, mais aussi comme le conflit belliqueux qui fit de l'Espagne un exemple de résistance nationale, un laboratoire privilégié de formules politiques non seulement théoriques -la Constitution de 1812-, mais aussi pratiques -la guerre de guérilla- devenant ainsi de vrais archétypes révolutionnaires et donnant une dimension clairement européenne à la guerre de l'indépendance espagnole. L'historiographie des vingt-cinq dernières années a clairement révélé que l'on ne peut plus considérer la guerre de 1808-1814 comme une lutte entre la réaction et la révolution ${ }^{4}$. Pourtant perdure encore le topique de Georges Lefebvre, dans son ouvrage fondamental sur Napoléon, selon lequel les Espagnols ne le combattirent pas comme un despote et un envahisseur, mais plutôt parce qu'il représentait à leurs yeux la Révolution et la perspective d'une modernisation. Le récent livre de Vinzenzo Criscuolo $^{5}$ se fait aussi l'écho de cette vision de Napoléon : il croyait que la résistance espagnole était comme celle des Vendéens ou des Sanfedistes napolitains. Il n'a pas compris le mouvement national patriotique que la minorité libérale espagnole avait tenté de consolider face à l'ennemi extérieur, ni son cri de « la patrie en danger » comme cela s'était produit pendant la Révolution française.

5 Pronunciamiento, comme liberal, guerrilla ou junta, sont des mots espagnols qui ont passé dans le langage universel. Mais le terme "pronunciamiento» a des connotations négatives pour l'histoire contemporaine de l'Espagne: il suffit de se souvenir du soulèvement du général Franco contre la Seconde République espagnole qui fut un vrai modèle de "pronunciamiento" militaire (et non pas un "coup d'État»). Mais la phénoménologie d'un événement qui a parcouru nos XIXe et XXe siècles ne doit pas 
occulter sa gestation ni le contexte dont il est issu qui lui donna un caractère clairement libéral et révolutionnaire très différent de celui des pronunciamientos militaires postérieurs; par conséquent, il est incorrect de parler de ces premiers pronunciamientos espagnols comme des " coups d'État militaires " ${ }^{6}$, ce qui pourtant est encore fréquent dans l'historiographie de la révolution libérale espagnole.

6 Après le soulèvement de 1808 , la guerre contre la France napoléonienne constituait une combinaison originale de guerre régulière et de guérillas. De cette double expérience naquit en 1814 une armée dotée de caractéristiques propres et singulières, une armée nouvelle en processus de transformation et, pour ce motif, profondément divisée. Soumise aux changements sociaux et idéologiques décisifs qu'avaient subis les armées européennes après les guerres de la Révolution française et de l'empire napoléonien, elle devint un instrument pour l'implantation de l'idéologie nationale ainsi qu'un véhicule de promotion sociale. L'intégration en une même institution de composants sociaux très divers, - comme le traditionnel, aristocratique, et le composant populaire qui se concentra dans les classes subalternes et de sous-officiers- était une caractéristique de cette armée espagnole surgie entre 1808-1814, après la faillite de l'institution de l'ancien régime face à l'invasion étrangère. La récente pénétration dans la nouvelle armée de secteurs provenant de la guérilla et la claire adhésion d'un secteur des commandements et des officiers à l'idéologie libérale de l'époque, donna un nouvelle tonalité nettement pro-constitutionnelle à une partie de l'armée. En même temps, le souvenir et l'expérience des techniques de la guérilla (avec son déploiement de noyaux dispersés et protégés par la distance et l'encerclement, en évitant les endroits occupés par de fortes garnisons ennemies, le choix de sites périphériques couverts par des forêts et des montagnes, la recherche de ressources naturelles pour se maintenir), exercèrent une forte influence sur tous ceux qui engagèrent des actions contre le gouvernement au cours du premier tiers du XIXe siècle.

7 Les élites libérales alertées par le potentiel subversif de la guerre de guérillas, conçurent le pronunciamiento comme une stratégie de lutte contre l'absolutisme qui ne recherchait le concours populaire qu'au moment où éclaterait une insurrection qui serait contrôlée par les chefs politiques et militaires et dont on attendrait l'appui, non pas de la campagne, mais des principales villes, la vraie base sociale du libéralisme; le pronunciamiento était un phénomène exclusivement urbain dont l'élément militaire constituait certainement la pièce fondamentale, l'appui nécessaire et l'instrument le plus adéquat pour la matérialisation du projet libéral. D'autre part, sans chefs militaires sur le front, on ne pouvait communiquer à la nation le message du pronunciamiento et le signal de l'insurrection. Ce furent les conditions de répression et de violence imposées par l'absolutisme espagnol qui obligèrent les libéraux à pratiquer la conspiration dans des associations ou «loggias" de caractère franc-maçonnique, bien qu'en Espagne ne soient implantés ni la franc-maçonnerie ni le carbonarisme et ce ne fut que lors du Trienio liberal de 1820-1823, que le libéralisme espagnol s'introduisit pleinement dans le monde des sociétés secrètes européennes de l'époque. Ne pouvant s'établir légalement, des «juntes» clandestines s'organisèrent, ce qui était précisément l'objectif du pronunciamiento : créer une conjoncture révolutionnaire permettant d'établir des juntes légales dans les provinces, puis une junte centrale qui puisse exercer les fonctions du gouvernement provisoire. Tel était le schéma de la révolution «juntiste », issue aussi de la guerre de l'indépendance et qui caractérisait la modalité de la révolution libérale espagnole. 
8 Il est important de préciser que, même si l'on parlait d'insurrection, le changement politique ne devait pas être nécessairement armé ni sanglant, mais simplement provoqué par une crise strictement politique. Il s'agissait d'une action en principe pacifique, d'une espèce de plébiscite insurrectionnel qui n'était pas en contradiction avec le recours aux armes en tant que mesure de défense contre l'éventuelle intervention de l'armée de Ferdinand, mais seulement pour protéger l'acte du pronunciamiento. Il faut insister sur le fait que le pronunciamiento ne prétendait pas déclencher la guérilla, ni affronter les forces absolutistes, mais provoquer l'insurrection des noyaux urbains en tant que forme de pression populaire sur le gouvernement et de soutien des «pronunciados ». Le véritable caractère de la stratégie politique du pronunciamiento provenait ainsi de sa subordination explicite à l'objectif politique auquel il était destiné : le renversement de l'absolutisme et l'implantation de la Constitution. Une stratégie qui pouvait ou ne pouvait pas triompher, mais qui réunissait tous les conditionnements et les règles requises. La séquence des faits était clairement établie: les libéraux devaient conspirer pour se "soulever» et ils "se soulevaient» pour provoquer une série d'effets politiques qui aboutiraient à la conquête du pouvoir pour restaurer la liberté. Le « geste » de rébellion du chef militaire qui initiait le pronunciamiento devait toucher par contagion toutes les périphéries urbaines espagnoles. Or, le bon fonctionnement du processus insurrectionnel réclamait une stratégie idéale, en circonférence, allant de la périphérie vers le centre du pays, faisant ainsi pression sur le gouvernement pour imposer sans lutte la finalité politique proposée. Cela impliquait à la fois l'existence et le développement de différents foyers insurrectionnels s'appuyant sur une force militaire qui permettrait aux « pronunciados » de se consolider en différents points. Il faut distinguer en outre le pronunciamiento principal des pronunciamientos locaux dont étaient responsables d'autres chefs militaires chargés de le « seconder ». La difficulté à matérialiser le pronunciamiento, et plus encore à assurer le succès de celui-ci, était évidente et une organisation patiente et laborieuse était nécessaire ainsi que la coordination des conspirateurs. La pratique de la conspiration autant que le point culminant du pronunciamiento étaient des activités minoritaires et sectaires car l'on avait seulement recours à l'appui populaire au moment où l'insurrection éclatait. La conception de la politique et de l'histoire soutenue par ces libéraux se fondait sur le principe selon lequel la vertu, les grands idéaux et la force mobilisatrice de l'exemple provoquaient l'adhésion immédiate du peuple au parti des leaders auto-proclamés. Leur messianisme révolutionnaire les maintenait dans la croyance que l'action héroïque de quelques hommes, même si elle ne les menait pas au triomphe auquel ils aspiraient, était nécessaire pour encourager et stimuler les efforts suivants. Une pureté de conviction qu'il faut insérer dans le contexte romantique de l'époque. L'importance de l'action, la culture des émotions, le risque, la valeur et l'exigence de libertés, le symbolisme qui accompagnait le rituel de l'initiation dans les sociétés secrètes, étaient des éléments de la vision romantique du monde partagée par un large secteur des élites sociales et politiques. La contrerévolution européenne de 1815 , avec ses séquelles de persécutions et d'exils, donna une unité et une identité collective à ce libéralisme qui avait le même langage et la même mentalité typiquement romantiques dans tous les domaines, y compris celui de la politique. Il est important de tenir compte des considérations précédentes pour comprendre le mécanisme du pronunciamiento: entre l'éclatement de celui-ci et la prise de pouvoir, il y avait un parcours destiné à les unir sans que l'on puisse garantir à l'avance son succès, qui constituait le véritable nœud de l'affaire. 
Dans le phénomène du pronunciamiento, il y a donc quatre éléments articulés entre eux comme des chaînons dont les deux premiers, conspiration et pronunciamiento, sont assurés par la détermination du groupe d'hommes décidés à agir. Le quatrième, la prise du pouvoir, est le résultat du bon fonctionnement de l'ensemble. Au milieu, se trouve la variable, la contagion insurrectionnelle en tant qu'effet du pronunciamiento, une véritable inconnue dont l'éclaircissement dans un sens ou un autre dépend à son tour de deux facteurs essentiels: la réponse du peuple et la réaction du pouvoir. L'interrelation profonde, mais pas mécanique, entre les quatre éléments signalés ainsi que l'indépendance relative de chacun d'eux, fut évidente en janvier 1820 lors de l'échec militaire du pronunciamiento de Riego à Cadix, ce qui n'empêcha pas mais provoqua malgré tout, au bout d'un mois, l'effet attendu: les insurrections urbaines provoquèrent le changement de régime et obligèrent Ferdinand VII à proclamer la Constitution de 1812 au mois de mars de la même année. Cette victoire des libéraux couronnait les multiples tentatives frustrées de pronunciamientos qui s'étaient produites presque une fois par an entre 1814 et 1820 . Les propres libéraux de l'époque affirmaient que leurs actions avaient été la cause principale de la chute d'un absolutisme harcelé par de graves problèmes financiers et structuraux ${ }^{7}$. Mais il n'en est pas moins vrai que le pronunciamiento de Riego signifia le triomphe de la révolution de 1820 et qu'il marqua, autant en Espagne qu'en Europe, un archétype révolutionnaire qui perdura jusqu'aux mouvements révolutionnaires de 1830, dont les éléments fondamentaux étaient: la conspiration dans des sociétés secrètes de type carbonaro, la mise en pratique du pronunciamiento insurrectionnel et l'implantation d'un régime représentatif comme objectif politique.

Le libéralisme espagnol, un idéal de liberté pour les libéraux européens des années 1820.

Le modèle de Riego comblait l'ardent désir de liberté des romantiques libéraux : depuis la guerre de l'Indépendance, la cause de l'Espagne fut l'étendard de la lutte contre l'absolutisme. C'est pourquoi le Trienio liberal espagnol (1820-1823) eut pour l'Europe libérale de l'époque une signification similaire à celle qu'aura la Seconde République espagnole pendant le XXe siècle. À partir de 1815, à mesure que se modifiait le climat politique grâce au triomphe de la Restauration et à la suprématie de la culture politique du libéralisme doctrinaire, le modèle de la Constitution espagnole de 1812 devint la référence politique qui alimentait les propositions pour une rupture avec l'absolutisme ainsi que la menace pour les libéraux modérés qui, comme ce fut le cas en France, considéraient que la révolution contre l'Ancien Régime était terminée. Ce premier libéralisme espagnol fut le véhicule par lequel les principes de la Révolution française passèrent en Europe, dynamisant les mouvements politiques libéraux, surtout les plus radicaux ${ }^{8}$. C'est la raison pour laquelle la Constitution de 1812 fut l'un des éléments de ce que j'ai défini comme l'archétype révolutionnaire dominant à cette époque, ses caractéristiques étant spécialement propres à imposer le système constitutionnel aux monarques absolus. G. Dufour a signalé à ce propos ${ }^{9}$ que la publication massive de la Constitution de Cadix avait permis aux Français de prendre à nouveau connaissance des principes révolutionnaires si lointains après l'Empire, l'Espagne devenant un modèle révolutionnaire différent du français, «c'est-à-dire un modèle révolutionnaire sans la Terreur dont le souvenir discréditait tellement la cause révolutionnaire abusivement qualifiée par ses adversaires de jacobine ». Cela explique l'immense intérêt suscité à cette époque par tout ce qui se référait à l'Espagne et à son histoire, le mot « libéral » 
servant à qualifier celui qui approuvait le pronunciamiento de Riego et le souhaitait pour la France où les tentatives de pronunciamiento se multiplièrent entre 1821 et 1822.

11 Ce premier libéralisme espagnol né à Cadix domina le premier tiers du XIXe siècle libéral européen, notamment en Europe latine grâce à son modèle constitutionnel qui permettait l'accès au pouvoir des élites et la transformation des institutions tout en évitant les radicalismes de la Révolution française. C'est ce qui se passa en Europe méridionale où existaient des problèmes communs dus aux effets produits par la double révolution française et industrielle dans le domaine de la propriété, la possession et la culture de la terre qui était toujours considérée comme la principale source de richesse dans des sociétés essentiellement agraires. L'affaiblissement ou l'abolition du féodalisme qui se produisirent pendant la période 1789-1814 signifia, en même temps que l'attaque aux biens communaux des villages et le désamortissement des terres de l'Église, un changement substantiel dans le secteur agraire, avec un important transfert de la propriété au bénéfice presque exclusif des nobles et des bourgeois ${ }^{10}$. Dans la plus grande partie de l'Europe latine, l'abolition du féodalisme était due à l'action des armées françaises d'occupation, sauf en Espagne où elle résulta des Cortès de Cadix. Mais depuis 1814, sauf en France et dans les régions ayant subi une profonde transformation en raison de leur ancienne incorporation à la France, comme c'était le cas du nord de l'Italie, les restaurations bourboniennes annulèrent l'application de ces principes abolitionnistes, comme en Espagne et au Portugal où la restauration fut moins importante parce qu'il y eut moins de transformations. Même en Italie, bien que les structures féodales aient été supprimées, les secteurs de la bourgeoisie étaient encore loin d'avoir un pouvoir politique. Du point de vue économique, elles se transformeraient après 1814, grâce à l'inadaptation de leurs structures sociales au développement du capitalisme qui avait de plus en plus tendance à rompre les cadres nationaux pour se convertir en un système mondial. C'est-à-dire que la propre expansion du capitalisme mondial utilisait les systèmes dans les pays où, sans ces influences externes, les contradictions n'auraient pas été aussi aiguës ni dénaturées, et n'auraient pas entraîné leurs gouvernements respectifs à une faillite financière. Ces difficultés économiques de l'absolutisme facilitèrent l'action politique de l'opposition libérale : des répercussions immédiates de la Révolution espagnole de 1820 s'ensuivirent dans toute l'Europe et Metternich la considéra comme pire que la française, celle-ci étant restée locale alors que l'espagnole se propagea en Europe ${ }^{11}$. Ce qui était vrai et, avec l'entrée en jeu des intérêts reliés aux colonies américaines, au lieu d'être européenne, la révolution devint mondiale.

Le plus grand impact de la révolution de Riego se situe surtout au Portugal et en Italie, car la France et l'Angleterre ainsi que les puissances légitimistes de l'Autriche et de la Russie utilisaient ces puissances de second rang comme marchés pour leurs produits industriels ou comme sources de matières premières. Le pronunciamiento libéral du 24 septembre 1820 au Portugal était directement inspiré du pronunciamiento de Riego et les libéraux espagnols lui prêtèrent appui. Le sentiment de l'» Alianza Ibérica " pénétra dans les secteurs les plus radicaux du libéralisme portugais. Certains d'entre eux préconisaient même un constitutionnalisme ibérien car ils voyaient l'union avec l'Espagne libérale comme un plus sûr moyen de se libérer à la fois de l'absolutisme portugais et du colonialisme anglo-brésilien ${ }^{12}$. Depuis 1826, ce furent les propres exilés espagnols qui demandèrent à l'empereur du Brésil, Don Pedro de Braganza, d'unir l'Espagne et le Portugal en une monarchie constitutionnelle, de caractère modéré, les libéraux espagnols étant convaincus que Ferdinand VII n'accepterait jamais un système 
représentatif, ce qui explique le paradoxe d'avoir déjà offert la couronne d'Espagne à son ancien ennemi, le frère de Napoléon, Joseph Bonaparte. L'Espagne fut aussi un modèle pour l'Italie, de 1808 à 1830 , comme l'ont souligné de nombreux travaux ${ }^{13}$. Pendant le Trienio liberal de 1820-1821, se réfugièrent dans la Péninsule Ibérique tous ceux qui étaient poursuivis par l'absolutisme, cristallisant alors les relations et les expériences communes de lutte, dont la toile de fond était l'internationalisme libéral, une caractéristique fondamentale du libéralisme de l'époque, bien que l'historiographie ne lui ait guère prêté l'attention que cela mérite ${ }^{14}$.

Une grande partie des libéraux européens, comme les Espagnols en exil ${ }^{15}$, n'acceptait pas les frontières imposées après 1815 contre l'ordre napoléonien. Ils furent obligés de réfléchir sur la relation entre leurs démarcations nationales respectives et la nouvelle Europe qu'ils imaginaient, ainsi que de se livrer à la recherche d'une unité supérieure des nations désunies permettant d'agir dans le cadre le plus libéral possible. La thématique européenne dominait la thématique nationale, ce qui était logique, étant donné que non seulement il fallait combattre un ordre politique social intérieur et oppressif mais aussi le système européen de la Sainte-Alliance ${ }^{16}$, défenseur de l'absolutisme et sa garantie dans les différents États de l'Europe méridionale.

L'existence de l'Empire avait permis d'unifier les luttes, alors que la mort de l'empereur à Sainte-Hélène ouvrait la voie au mythe du héros romantique auquel les libéraux ne purent se soustraire, notamment les militaires, bien qu'ils en critiquaient le despotisme à l'égard des peuples. Le patriotisme libéral romantique s'unit au phénomène culturel et à la conspiration du libéralisme insurrectionnel espagnol, qui reprit les valeurs militaires de l'époque napoléonienne, remodelées par la suprématie du civil et par la conception romantique du rôle du héros dans l'histoire. Deux générations européennes, proches dans le temps et dans leurs idéaux, s'unirent pour participer ensemble à l'expérience napoléonienne, bien que certains eussent lutté contre Napoléon comme ce fut le cas des militaires libéraux espagnols ${ }^{17}$. Cette dimension internationaliste du libéralisme, prit force pendant les crises révolutionnaires, aussi bien pendant celle de 1823 que pendant celle de 1830 . Ce fut surtout en 1823 , lors de la défense du régime libéral espagnol, que carbonari et bonapartistes s'allièrent en formant un réseau de relations, qui existaient déjà dès 1822, entre Londres, Lisbonne, Paris et le Midi français, Barcelone et Madrid, disposés à affronter les troupes du duc d'Angoulême qui envahirent l'Espagne le 7 avril 1823. Il y avait d'importants libéraux italiens (certains avaient même été combattants dans l'armée impériale et étaient entrés dans la résistance espagnole pendant le conflit qui débute en 1808), ainsi que des Portugais, des Anglais et des Français. Le Calabrais Guglielmo Pepe, ancien soldat de l'armée impériale qui avait lutté pendant la guerre de l'Indépendance espagnole, pour coordonner l'action de ces libéraux , créa une société de carbonari qui perdura pendant les années vingt s'appelant initialement "Régénérateurs européens » ${ }^{18}$. D'autre part, le gouvernement libéral espagnol groupa, non sans certaines résistances de la part de modérés, les combattants étrangers dans les dénommées Légions libérales étrangères par un décret des Cortès du 30 avril 1823. Parmi ces combattants, se distingue le militaire britannique Robert Wilson, déjà décoré auparavant au Portugal pour son aide à la cause ibérienne et qui passe de la Galicie au Portugal en 1823 et du Portugal à Cadix, puis est obligé de retourner dans son pays par Gibraltar.

15 Néanmoins, à un niveau interne, la résistance libérale espagnole devant l'invasion française de 1823 ne peut être comparée avec la mobilisation qui eut lieu en 1808, car 
les divisions internes et le malaise social engendré par la politique économique du libéralisme au pouvoir provoquèrent la passivité de la plus grande partie de la population majoritairement paysanne. Ce ne fut pas le cas dans les principaux noyaux urbains où le libéralisme avait pénétré à fond pendant la courte existence du régime libéral du Trienio : ceux-ci résistèrent jusqu'au bout, comme la Catalogne et Carthagène, en prolongeant la mal nommée, par le gouvernement français, "Guerre d'Espagne » jusqu'au mois de novembre $1823^{19}$.

16 La défaite subie par les régimes libéraux établis en 1820 en Espagne, au Portugal et en Italie, condamna à l'exil d'importantes personnalités politiques et intellectuelles de ces pays, parmi lesquels prédominaient les militaires libéraux. Malgré les changements de toutes sortes opérés dans une société en transformation comme la société européenne pendant les années 1820-1830, l'émigration politique militante continua à agir dans les termes du modèle forgé par la révolution espagnole de 1820. Il est vrai que le libéralisme insurrectionnel espagnol perdait sa force opérationnelle en tant qu'élément politique face à d'autres causes comme l'indépendance de la Grèce ou bien, depuis 1831, celle de la Pologne, alors que la Constitution belge de 1831 relégua l'espagnole de 1812. Mais la stratégie basée sur le pronunciamiento, ne pouvait être déjà considérée comme annulée en tant qu'expérience historique de la lutte libérale. Elle le serait après la révolution française de 1830 qui réorganisa le panorama international européen et introduisit des changements décisifs dans l'organisation politique et sociale des mouvements révolutionnaires. Cependant, avant les événements de 1830 et leurs répercussions, en raison de l'intervention décisive des grandes puissances dans l'échec des révolutions de 1820 , le camp anti-absolutiste acquit la conviction que seul un changement dans la corrélation de forces de la politique européenne en faveur du mouvement libéral, pouvait favoriser la révolution dans les pays respectifs : le succès d'une révolution nationale semblait conditionné par la situation internationale. C'est ainsi que naquit un mouvement patriotique européen dont la cause devint celle des Espagnols, des Italiens, des Portugais, des Français et des indépendantistes latinoaméricains, du peuple grec et des décembristes russes. L'idée d'une Europe fédérale, ou d'États-Unis d'Europe, se répète constamment dans les textes de l'époque, en formules qui furent expliquées par la suite, mais qui correspondent au contexte historique de l'exil libéral européen des années vingt et à la philosophie politique aussi bien des libéraux modérés que des démocrates. Il s'agissait de concilier le particularisme avec l'universalisme pour obtenir une coexistence pacifique entre les nations et de rendre les petits pays plus forts.

17 Cosmopolitisme, fédéralisme et patriotisme n'étaient pas des termes incompatibles à cette phase de la construction de nouvelles nations. Il s'agissait de fonder un nouvel ordre politique et intellectuel alternatif à la fois au cosmopolitisme du XVIIIe siècle, au modèle de l'Empire napoléonien, mais aussi à celui d'un patriotisme étroit, renfermé dans chaque patrie en particulier. L'exil européen des années 1820 constitue ainsi un phénomène culturel de haute importance compte tenu de l'échange entre les courants d'idées qui rendirent propice l'émigration politique d'où surgit progressivement une société intellectuelle européenne. Dans le domaine de l'action, l'unanimité en tant que point de départ de référence à la stratégie du pronunciamiento, permettait de concilier des programmes politiques divers qui allaient du libéralisme modéré à la démocratie, en laissant la discussion sur les formes de gouvernement pour après le changement politique. Les fissures idéologiques ne se manifestèrent clairement qu'après la révolution française de 1830 et le triomphe des révolutions libérales en Espagne et au 
Portugal pendant les années 1830. Jusqu'alors, les libéraux espagnols, qui n'étaient pas révolutionnaires par principe, n'avaient pu faire une claire distinction, comme une grande majorité l'aurait voulu, entre le libéralisme et la révolution à laquelle ils furent acculés par l'absolutisme responsable d'avoir plongé le pays dans une succession de guerres, de répressions et d'exils. Les tentatives de pronunciamiento se succédèrent jusqu'en 1831, et bien qu'elles ne réussirent guère à abolir le système en Espagne, elles l'affaiblirent, contribuant ainsi à sa chute après la mort de Ferdinand VII, en septembre 1833. La mémoire historique continue à perpétuer les trois mythes du libéralisme du XIXe siècle espagnol qui personnifient ce libéralisme insurrectionnel auquel nous avons consacré ces pages: les militaires Riego et Torrijos ainsi que l'héroïne de Grenade, Mariana de Pineda, victimes du martyrologe du règne de Ferdinand et que l'on actualise toujours dans des situations de crise institutionnelle ou les conjonctures révolutionnaires de l'histoire d'Espagne. Leur exemple prouva à toute l'Europe que la liberté était le bien le plus précieux.

\section{NOTES}

1.Ce travail s'inscrit dans le projet de recherche BHA 2001-2509 du PNICDIT.

2.C'est ce que remarque Salvo Mastellone, Storia ideologica d'Europa da Sieyès a Marx, Bologne, Sansoni, 1974, pp.181-182.

3.La théorisation des pronunciamientos libéraux du règne de Ferdinand VII a été réalisée à partir de la recherche sur les pronunciamientos de ladite " décennie abominable » (1823-1833), et se trouve dans mon livre La utopía insurreccional del liberalismo. Torrijos y las conspiraciones de la década ominosa (1823-1831), Crítica, Barcelone, 1989.

4.Pour une réflexion critique en rapport avec ce sujet, sur les travaux de l'hispanisme français, voir mon article « El hispanismo francés desde la historiografía espagnola: ¿Francia, revolución: España, reacción? », dans Ayer, n 31 (1998) (Ismael Saz, ed. España : La mirada del otro), pp. 43-57.

5.Vinzenzo Criscuolo, Napoleón, Alianza Editorial, Madrid, 2000 (première édition en italien en 1997).

6.Eric J. Hobsbawn, dans Revolucionarios, Barcelona, Ariel, 1978, p. 110, souligne clairement la différence entre les pronunciamientos espagnols de la première moitié du XIXe siècle, en étroite relation avec la politique libérale, et ceux de la deuxième moitié du même siècle lorsqu'ils deviennent « une entreprise d'affaires spéculatives pour les généraux », et par rapport aux pronunciamientos du XXe siècle, qui « cessèrent d'avoir une relation quelconque avec le libéralisme ».

7.Joseph Fontana, dans son ouvrage La Quiebra de la Monarquía Absoluta, publié il y a plus de 30 ans et réédité par Crítica, Barcelone, en 2002, établit le modèle de crise de l'Ancien Régime espagnol, depuis l'analyse des problèmes financiers de l'État absolutiste.

8.Pour la projection internationale de la Constitution de 1812, voir Ignacio Fernandez Sarasola, « La Constitución española de 1812 y su proyección europea e iberoamericana ", dans Fundamentos: Modelos constitucionales en la historia comparada, 
2/2000, pp. 359-440. Sur l'influence française sur les Cortès de Cadix et la Constitution de 1812, voir un récent état de la question de son traitement historiographique en Espagne, par Jean René Aymes, « Le débat idéologique et historiographique autour des origines françaises du libéralisme espagnol: Cortès de Cadix et Constitution de 1812 », dans la revue électronique Historia Constitucional, $\mathrm{n}^{\circ} 4$ (juin 2003), Área de Derecho constitucional, Universidad de Oviedo.

9.Gérard Dufour, « El primer liberalismo español y Francia », dans Emilio La Parra et Germán Ramirez (éd.), El primer libéralismo: España y Europa, una perspectiva comparada(Foro de Debate), Valence, Biblioteca Valenciana, 2003, pp. 125-136. 10.Salvo Mastellone, Storia ideologica d'Europa (...), op. cit., pp. 182-187.

11.Cité par Josep Fontana dans « Per què van envair Espanya els Cent Mil Fills de Sant Lluís? », Recerques, n 19, 1979, pp. 17-33.

12.J'ai traité ce sujet sous l'angle des relations entre libéraux espagnols et portugais dans « Los orígenes del iberismo (1815-1832) », Homenaje a Josep Fontana, Crítica, Barcelone, 2004.

13.L'un des derniers parus : Vitorio Scotti Douglas, « El libéralismo español en Italia: un modelo de corta duración », dans Emilio La Parra et Germán Ramire (éd.), El primer libéralismo, op. cit., pp. 315-340. Également, Agostino Bistarelli, « Vivere il mito spagnolo. Gli esiliati italiani in Catalogna durante il Trienio Libéral » (I-II), Trienio, 32 (1998), pp. 5-14, et 33 (1999), pp. 65-90.

14.J'insiste sans cesse, depuis longtemps, sur cette ligne de travail depuis mon article "Constitucionalismo, estrategia insurreccional e internacionalismo liberal en la lucha contra el Antiguo Régimen español (1823-1831) », Revista de História das Ideias, $\mathrm{n}^{\circ} 10$ (1988), pp. 485-506. Je me suis occupée aussi de cette dimension internationaliste du libéralisme européen, dans « La opinión liberal ante la invasión francesa: Cataluña, $1823 »$ : Sociabilidad y Libéralismo en la España del siglo XIX. Homenaje a A. Gil Novales, Milenio, Lleida, 2001, pp. 147-156. Les jeunes chercheurs prêtent actuellement une plus grande attention à ce thème, comme le démontrent les travaux de Christiana Brennecke : «Internacionalismo liberal, romanticismo y sed de aventura. La oposición inglesa y la causa de España en los años veinte del siglo XIX ", Segon Congrés Recerques.

Enfrontaments civils: postguerres i reconstruccions, Universita de Lleida, Lleida, 2002, vol. I, pp. 459-474, et du même auteur, «Sir Robert Wilson y el nacimiento de las Legiones Libérales Extranjeras durante el Trienio Liberal (1820-1823)»; communication présentée au Congrès international : Orígenes del liberalismo. Universidad, Política, Economía. Université de Salamanque 1-4 octobre 2002, qui se trouve dans le CD contenant les communications de ce Congrès.

15.Sur l'exil des années vingt, voir l'analyse et l'état de la question de Juan Francisco Fuentes Aragones, « Procedencia y destino geográfico del exilio liberal español en la década ominosa », dans Segon Congrés de Recerques, op. cit. pp. 542-553.

16.Christophe Charle, Les intellectuels en Europe au XIXe siècle, Paris, Seuil, 1996, pp. 113-122.

17.J'ai souligné ces questions dans ma biographie du militaire espagnol: « José María Torrijos(1791-1831): Conspirador romántico », dans Isabel Burdiel/Manuel Perez Ledesma (dir.), Liberales, agitadores y conspiradores, Madrid, Espasa Calpe, 2000, pp. 73-98. 18.Les fonds documentaires où j'ai consulté les sources qui me permettent de faire ces affirmations se trouvent dans les Archives départementales des Pyrénées-Orientales, Perpignan, Série M, Sous-série 4M.Police ; dans les Archives Administratives de la Guerre, Paris: « Armée de l’Espagne,1821-1828 », et dans les Arquivos Nacionais da 
Torre do Tombo, Lisbonne, « Correspondencia Legaçoes portuguesas en Espanha.

Legaçâo Portugal em Madrid », dans l'inventaire du Ministeiro de Negócios

Estrangeiros.

19.Sur l'occupation française en l'Espagne, Gonzalo Butron, La ocupación francesa de España (1823-1828), Cadix, Université de Cadix, 1997.

\section{RÉSUMÉS}

Dans cet article, nous essayons d'expliquer le modèle révolutionnaire surgi en Espagne entre 1814 et 1820. Il se basait sur la conspiration dans des sociétés secrètes pour préparer le pronunciamiento insurrectionnel, dont l'objectif politique était l'implantation de la Constitution espagnole de 1812 par laquelle les principes de la Révolution française se propagèrent en Europe. Nous décrivons comment ce modèle de lutte contre l'absolutisme fut utilisé par les libéraux européens jusqu'aux révolutions de 1830, ainsi que son impact durant l'Europe de la Restauration.

Spanish Insurrectionary Liberalism (1814-1830)

In this article, an attempt is made to explain the revolutionary model that emerged in Spain between 1814 and 1820. It was based on conspiracy within secret societies preparing the insurgency pronunciamiento, the political goal being the implantation of the Spanish Constitution of 1812 promoting the principles of the French Revolution in Europe. An account is given of how this model of struggle against absolutism was used by European liberals up until the revolutions of 1830 , and also its impact during the Restoration period in Europe.

\section{INDEX}

Mots-clés : Espagne, insurrection, libéralisme, révolution libérale espagnole, pronunciamiento, internationalisme libéral

\section{AUTEUR}

IRÈNE CASTELLS OLIVAN

Universitat Autònoma de Barcelone 